\title{
Wetting transitions in polydisperse fluids
}

\author{
Matteo Buzzacchi, ${ }^{1}$ Nigel B. Wilding, ${ }^{1}$ and Peter Sollich ${ }^{2}$ \\ ${ }^{1}$ Department of Physics, University of Bath, Bath BA2 7AY, United Kingdom. \\ ${ }^{2}$ King's College London, Department of Mathematics, Strand, London WC2R 2LS, United Kingdom.
}

(Dated: August 22, 2017)

\begin{abstract}
The properties of the coexisting bulk gas and liquid phases of a polydisperse fluid depend not only on the prevailing temperature, but also on the overall parent density. As a result, a polydisperse fluid near a wall will exhibit density-driven wetting transitions inside the coexistence region. We propose a likely topology for the wetting phase diagram, which we test using Monte Carlo simulations of a model polydisperse fluid at an attractive wall, tracing the wetting line inside the cloud curve and identifying the relationship to prewetting.

PACS numbers: 64.70Fx, 68.35.Rh
\end{abstract}

Complex fluids such as colloidal dispersions, polymers and liquid crystals are inherently polydisperse, that is their constituent particles exhibit continuous variation in terms of some attribute such as their size, shape or charge. The presence of polydispersity in these systems can profoundly influence their thermodynamic and processing properties [1-3]. Recent work has shown that the bulk phase behavior of polydisperse fluids can be surprisingly rich, exhibiting novel features such as polydispersity-induced phase transitions in polymers [4], reentrant melting in colloids [5], and a sensitivity of coexistence properties to the presence of rare large particles [6]. This richness is traceable to fractionation effects [3]: at coexistence the distribution of the polydisperse attribute varies from phase to phase.

Notwithstanding the progress in elucidating the role of polydispersity in determining bulk phase behaviour, little is known regarding its effects on the wetting behavior of complex fluids. Besides being a matter of great fundamental interest (see e.g. [7-9]) an understanding of this behaviour is crucial for technological applications such as spin coating and ink-jet printing of polymer or colloidal films for devices and displays [10].

This Letter broaches the subject of wetting in polydisperse fluids. Starting from heuristic arguments based on the restricted case of a binary mixture, we propose likely wetting scenarios for polydisperse mixtures. These we test using detailed Monte Carlo simulations of a model fluid at an attractive wall. Our results reveal a novel form of wetting transition occurring inside the coexistence region, which is driven by changes in density and/or temperature, rather than by temperature alone as in the case of monodisperse systems. We study the locus of wetting transitions in the phase diagram and how it depends on the strength of the wall-fluid attraction. For strongly attractive walls we further identify a prewetting transition within the stable vapor phase region.

The state of a polydisperse system (or any of its phases) is described by a density distribution $\rho(\sigma)$, with $\rho(\sigma) d \sigma$ the number density of particles in the range of $\sigma \ldots \sigma+d \sigma$; for e.g. size polydispersity, $\sigma$ would be the particle diameter. In the most commonly encountered experimental situation, the form of the overall or "parent" distribution, $\rho^{0}(\sigma)$, is fixed by the synthesis of the fluid, and only its scale can vary depending on the proportion of the sample volume occupied by solvent. One can then write $\rho^{0}(\sigma)=n^{0} f^{0}(\sigma)$ where $f^{0}(\sigma)$ is the normalized parent shape function and $n^{0}=N / V$ the overall particle number density; as the latter is varied, $\rho^{0}(\sigma)$ traces out a "dilution line" in density distribution space. The phase diagram is then spanned by $n^{0}$ and the temperature $T$.

Phase separation in a polydisperse system occurs within a region of $n^{0}$ and $T$ delineated by the cloud curve, which records the temperature $T$ where phase separation first occurs for given $n^{0}$. Quite generally, one finds that at two phase coexistence the parent $\rho^{0}(\sigma)$ will split into two "daughter" phase distributions $\rho^{1}(\sigma)$ and $\rho^{2}(\sigma)$ with different shapes - this is the phenomenon of fractionation. The daughter distributions are related to the parent via a simple volumetric average: $(1-\xi) \rho^{1}(\sigma)+\xi \rho^{2}(\sigma)=\rho^{0}(\sigma)$, where $1-\xi$ and $\xi$ are the fractional volumes of the two phases. In contrast to a monodisperse system where the densities of the coexisting phases are specified by the binodal and depend solely on temperature, full specification of the coexistence properties of a polydisperse system requires not only knowledge of the cloud curve, but also the dependence of $\xi, \rho^{1}(\sigma)$ and $\rho^{2}(\sigma)$ on $n^{0}$ and $T$ [11].

To understand the effects of polydispersity on wetting transitions, we turn first to the simplest case of a binary mixture with densities $\rho_{1}$ and $\rho_{2}$ of two species of particles of different sizes; these are the analog of $\rho(\sigma)$. Fig. 1(a) sketches an exemplary [12] isothermal cut through a bulk phase diagram, showing a region of liquid-vapor phase separation with tie-lines that shrink to zero at a critical point $(\mathrm{CP})$. We assume that the particle-wall interaction strength (averaged over the two species) is sufficient that there is a finite region of coexistence, bordering the $\mathrm{CP}$, for which the liquid wets the wall (the wall is always wet at criticality because of the rapid decrease of the liquid-vapor surface tension [13]); a prewetting line extending into the one-phase region is also indicated.

The dilution line constraint of a fixed shape for $\rho^{0}(\sigma)$ reduces in the bidisperse case to a fixed ratio $\rho_{1}^{0} / \rho_{2}^{0}$. As $n^{0}=\rho_{1}^{0}+\rho_{2}^{0}$ is increased from zero, the system follows the dilution line into the coexistence region. For a given point 

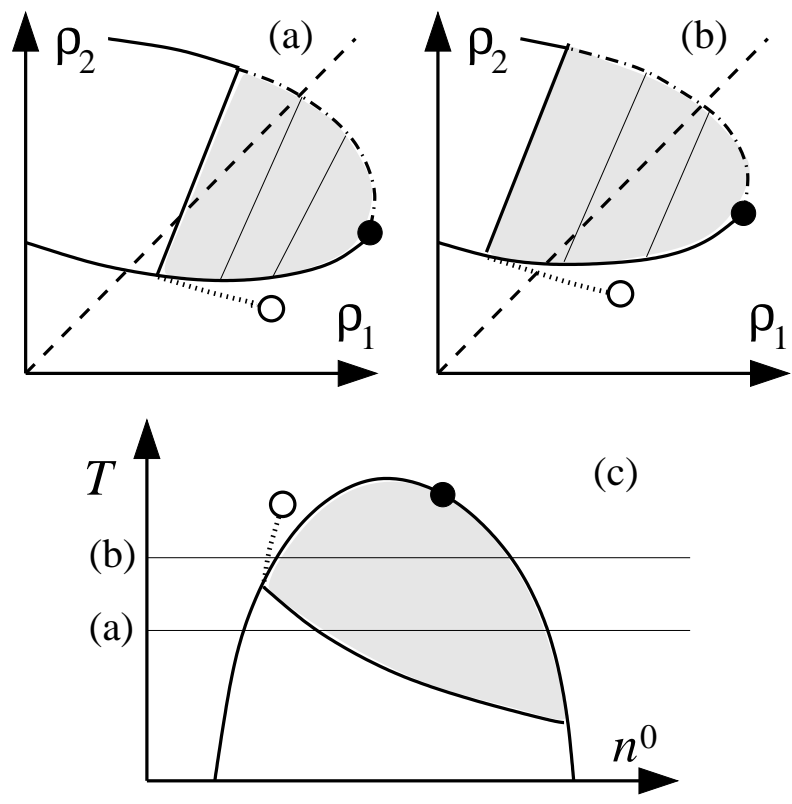

FIG. 1: Schematic phase diagram of the binary mixture described in the text. Thick solid line: coexistence boundary; thin solid lines: tie-lines; grey areas: liquid (dash-dotted line) wets wall; dashed line: dilution line; dotted line: prewetting line, with CP (open circle). Full circle: bulk CP. (a) and (b): isothermal cuts at two different $T$ as indicated in the full phase diagram (c) for fixed $\rho_{1}^{0} / \rho_{2}^{0}$.

on the dilution line, the parent splits into two daughter phases located at the ends of the tie-line which intersects this point; owing to fractionation, the daughters lie off the dilution line. In the example of Fig. 1(a), one sees that as the system enters the coexistence region the liquid does not initially wet the wall, but as $n^{0}$ increases further, the properties of the daughter phases change, and it starts to do so; crucially, this change occurs within the coexistence region. Fig. 1(b) shows a possible scenario at a higher temperature; here the phase coexistence region has shrunk and thus the dilution line now crosses the prewetting line in the one-phase region, indicating that a prewetting transition will occur on increasing $n^{0}$. In this case, the liquid daughters always wet the wall, and so no further transitions occur inside the coexistence region. Generalizing from these two fixed temperatures to a full $n^{0}-T$ phase diagram, we infer the topology shown in Fig. 1(c); the wetting transitions inside the coexistence region will occur for a range of temperatures and so define a wetting line which we expect to link the low and high density sides of the coexistence region. Provided this wetting line is first order where it intersects the gas branch, it will connect to a prewetting line, as shown.

We have not, of course, explored all possible phase diagram structures here; even for bidisperse systems at constant $T$, other possibilities could be envisaged. We are also not aware of any rigorous argument that indicates that the wetting line must have a negative slope as in our illustration. However, at least for weak wall interactions, where the wetting line is expected to enclose only a small region around the $\mathrm{CP}$, its slope should follow that of the cloud curve there; in all the examples of which we are aware, this slope is negative, and so we may anticipate that the scenario we have outlined is quite general.

To test the above ideas in a genuinely polydisperse setting, we have deployed Monte Carlo simulation to study a model fluid in which the polydisperse attribute $\sigma$ is the particle diameter. Particles interact via a Lennard-Jones potential of the form:

$$
u_{i j}=\epsilon_{i j}\left[\left(\sigma_{i j} / r_{i j}\right)^{12}-\left(\sigma_{i j} / r_{i j}\right)^{6}\right]
$$

with $\epsilon_{i j}=\sigma_{i} \sigma_{j} \epsilon / \bar{\sigma}^{2}$, where $\epsilon$ and $\bar{\sigma}$ set our energy and length scale units respectively, while $\sigma_{i j}=\left(\sigma_{i}+\sigma_{j}\right) / 2$ and $r_{i j}=\left|\mathbf{r}_{i}-\mathbf{r}_{j}\right|$. The potential was truncated for $r_{i j}>2.5 \sigma_{i j}$ and no tail corrections were applied. The diameters $\sigma$ are drawn from a (parental) Schulz distribution $f^{0}(\sigma) \propto \sigma^{z} \exp [(z+1) \sigma / \bar{\sigma}]$, with mean diameter $\bar{\sigma}$. We chose $z=50$, corresponding to a moderate degree of polydispersity: the standard deviation of particle sizes is $\delta \equiv 1 / \sqrt{z+1} \approx 14 \%$ of the mean. The distribution $f^{0}(\sigma)$ was limited to within the range $0.5 \bar{\sigma}<\sigma<1.4 \bar{\sigma}$.

The bulk liquid-vapor phase behaviour of the model of eq. (1) has previously been obtained [6] within the grand canonical ensemble (GCE) using bespoke computational techniques designed to ensure that the ensemble averaged density distribution always equals a desired parent form, by controlling an imposed chemical potential distribution $\mu(\sigma)$. Also implemented was a recently proposed strategy [11] which ensures that the two phases appear with equal probabilities in the GCE simulations. This results in finite-size corrections to the limiting bulk properties which are exponentially small in the system size - a feature which, it transpires, is crucial for accurately obtaining the wetting properties corresponding to a semi-infinite system. The study of ref. [6] yielded (inter alia) accurate estimates for the cloud curve and the form of $\mu(\sigma)$ at all points within the coexistence region. The CP was found to be located not at the temperature maximum of the cloud curve, but at higher density and lower temperature, as is also observed in experimental studies of polydisperse systems [14].

Equipped with knowledge of the bulk phase behavior of our model, we turn now to address its wetting properties [13] by introducing a single attractive wall to our system. Our simulation box has dimensions $L_{x} \times L_{y} \times L_{z}$, with $L_{x}=L_{y}=15 \bar{\sigma}$ and $L_{z}=30 \bar{\sigma}$ or $L_{z}=40 \bar{\sigma}$, and is periodic in the $x$ and $y$ directions. A fluid particle interacts with the attractive wall at $z=0$ via a potential

$$
U_{i w}=\epsilon_{i w}\left[\left(\sigma_{i w} / z_{i w}\right)^{10}-\left(\sigma_{i w} / z_{i}\right)^{4}\right]
$$

with $\epsilon_{i w}=\epsilon_{w} \bar{\sigma} \sigma_{i} / \bar{\sigma}^{2}$ and $\sigma_{i w}=\left(\sigma_{i}+\bar{\sigma}\right) / 2$. The wall at $z=L_{z}$ is completely hard.

In a canonical setting, the wall is "wet" at a given coexistence state point if the free energy is minimized when liquid is in contact with the wall, rather than gas. 
In our GCE simulations, we locate wetting points for a given wall strength $\epsilon_{w}$ by imposing the $\mu(\sigma)$ appropriate to some prescribed bulk values of $n^{0}$ and $T$; this corresponds to the conditions of a semi-infinite geometry. We then record the ensemble averaged density profile $\bar{n}(z)=\int d \sigma \bar{\rho}(z, \sigma)$ and the probability distribution $p(n)$ of the fluctuating total density $n=\int d \sigma d z \rho(z, \sigma)$; both of these are accumulated as histograms, one over $z$ and the other over $n$. We repeat these measurements, for a range of bulk state points in which $n^{0}$ is increased isothermally from the stable vapor region to values within the bulk coexistence region. Formally, wetting occurs within the GCE when the adsorption $\Gamma\left(n^{0}\right)=\int_{0}^{\infty}\left(\bar{n}(z)-n_{v}\right) d z$ diverges, with $n_{v}$ the vapor density. For systems of finite size, the wetting transition is identifiable as the value of $n^{0}$ at which the instantaneous profile $n(z)$ first becomes freely fluctuating. This leads to a linear decay of the $\bar{n}(z)$ along the $z$-direction; it is also signalled by the disappearance of a "vapor peak" in the distribution of the overall density $p(n)[15]$.

As an independent consistency check on this approach for determining wetting points, we have, for a broad selection of our state points, also implemented the distinct method described in ref. [16]. Here direct measurements (in geometries different to that considered above) of liquid-vapor, vapor-wall and liquid-wall surface tensions, together with Young's equation, $\gamma_{L V} \cos \theta=\gamma_{V W}-\gamma_{L W}$, serve to estimate the contact angle $\theta$. Wetting points follow as those combinations of $n^{0}$ and $T$ where $\theta$ vanishes. The requisite surface tensions were extracted from the form of $p(n)$ (via multicanonical simulations [17]) for both a bulk (periodic) system and a system with two identical attractive walls (at $z=0$ and $z=L_{z}$ ) as described in ref. [16]. In all cases, good agreement was found with the estimates of wetting points obtained from the observations of $\bar{n}(z)$ and $p(n)$, described above, indicating that the effect of the hard wall at $z=L_{z}$ on the freely fluctuating form of $\bar{n}(z)$ does not engender significant finite-size corrections to estimates of wetting points.

Fig. 2 shows our measurements of the locus of wetting transitions in the $n^{0}-T$ plane, together with the cloud curve, for three choices of the wall strength $\epsilon_{w}$. Considering first the weakest wall, $\epsilon_{w}=1.04$, we find that the line of wetting points traverses the coexistence region, intersecting the cloud curve discontinuously at its high and low density branches. The line has a negative gradient and encloses a portion of the coexistence region containing the $\mathrm{CP}$. For coexistence points inside this region the wall is wet, while for points outside, it is dry. Fig. 3 shows density profiles $\bar{n}(z)$ for two state points slightly on either side of the wetting line. When the wall is dry, the profile is gas like, except for a small enhancement at the wall. However, when the wall is wet, the liquid-gas interface fluctuates freely and the ensemble averaged profile is sloped because the maximum extent of the fluctuations is limited by the hard wall at $z=L_{z}$. For a system infinite in the $z$ direction, the time averaged profile of the wet wall would become flat as a function of $z$. We have veri-

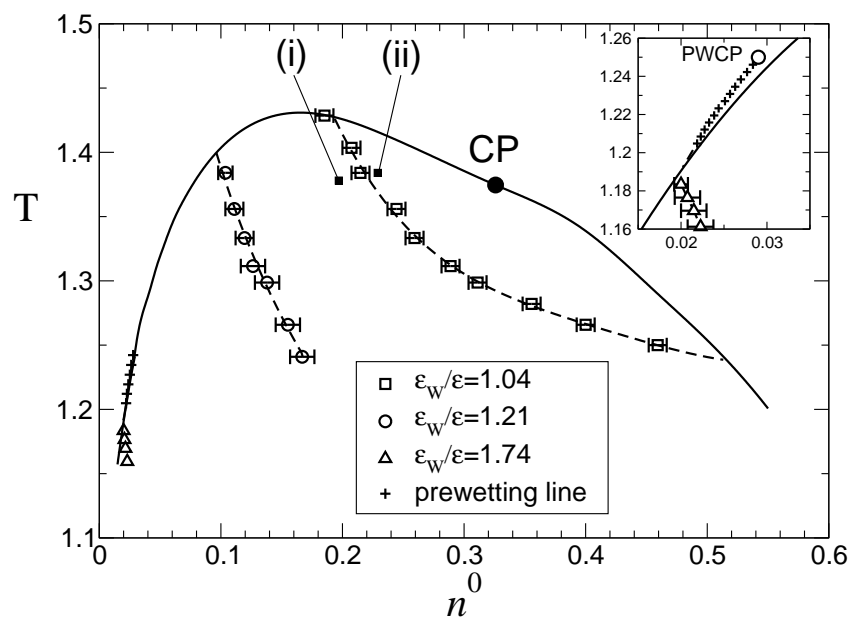

FIG. 2: Wetting phase diagram for three wall strengths. Full line: cloud curve obtained in Ref. [6]. Symbols: measured points on the wetting line; dashed lines guide the eye; small solid squares: state points referred to in Fig. 3. Inset: prewetting line observed for $\epsilon_{w} / \epsilon=1.74$.

fied that the profile indeed becomes flatter as we increase $L_{z}$ (inset Fig. 3), although no accompanying systematic changes in the locus of the wetting line were observed.

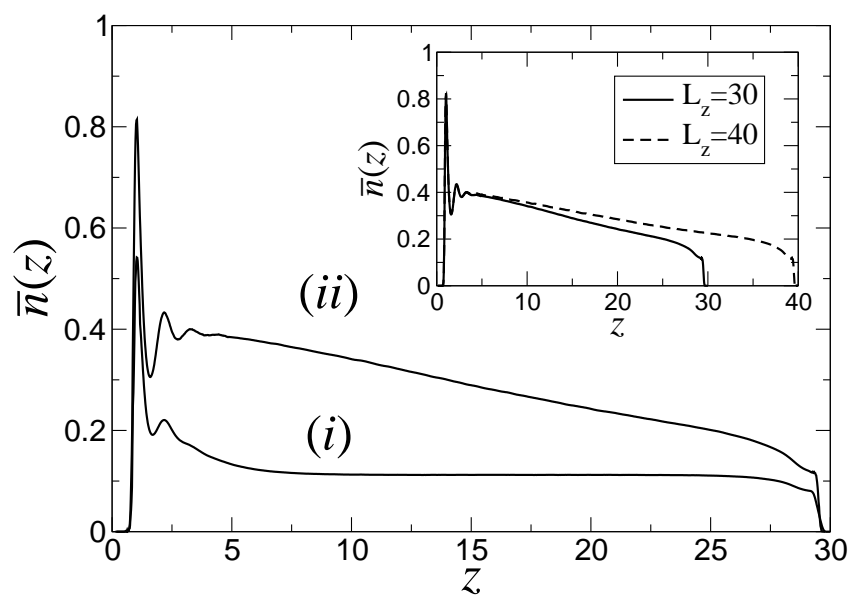

FIG. 3: Profiles $\bar{n}(z)$ for the state points indicated in Fig. 2, see text. The inset shows the effect of increasing $L_{z}$ on the profile of the freely fluctuating film.

For the intermediate wall strength $\epsilon_{w}=1.21$, the region of coexistence for which the wall is wet appears to be larger than for the weak wall, although we were unable to follow the wetting line right across the coexistence region due to the computational burden of simulating at very low $T$ and large $n^{0}$. For both the weak and intermediate wall strengths, no sign of prewetting was discernible in the stable vapor phase region. However a clear signature of prewetting was found for the strongest wall strength, $\epsilon_{w}=1.74$, at low $T$ in the stable vapor phase region near where the wetting line (of which we were able to determine only a small segment) intersects the gas branch of 
the cloud curve. The inset of Fig. 2 depicts the measured line of prewetting transitions, obtained from the points at which the film thickness jumps from a thin to thick finite value, as shown in Fig. 4. We note that the prewetting line appears to intersect the cloud curve tangentially, as is mandated thermodynamically [18].

Although the prewetting line represents a true (surface) phase transition, we note that no fractionation effects (such as splitting of the prewetting line into separate cloud and "shadow" curves - see Ref. [3]) are expected because neither of the coexisting phases are 'macroscopic' in the sense that their volume scales like the system volume. We further note that the observation of prewetting is evidence of a first order wetting transition [13], while its absence at smaller wall strengths could indicate that critical wetting occurs here. Intriguingly, there seem no grounds to rule out, a priori, a scenario in which the wetting transition changes from first order to continuous wetting at some point along the wetting line, the two regimes being separated by a tricritical wetting point.

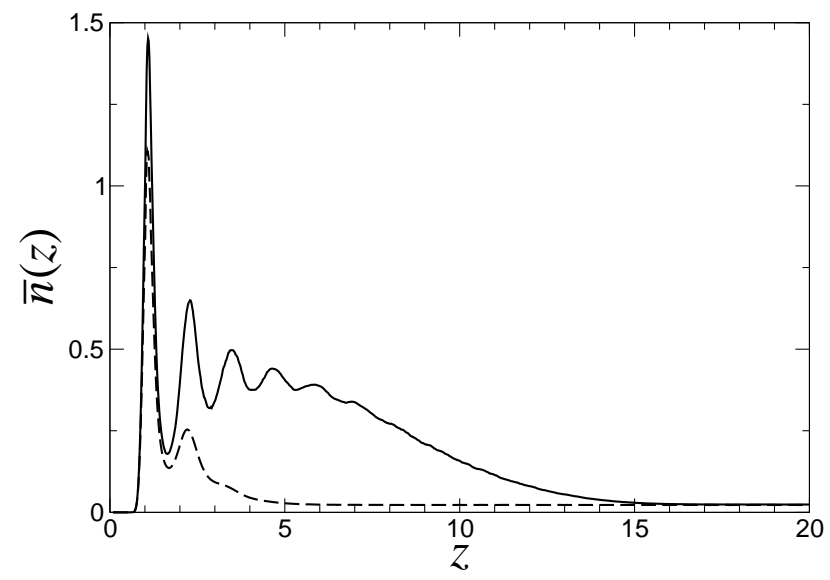

FIG. 4: Typical profiles $\bar{n}(z)$ for showing coexisting thin and thick films at the prewetting transition for $L_{z}=30 \bar{\sigma}$, see text.

The new feature of the wetting phase diagram of Fig. 2, compared to its monodisperse counterpart, is the occurrence of wetting transitions at constant $T$ driven by changes in parent density. The wetting line delineates a subregion of the coexistence region for which the wall is wet, and which contains the critical point. We expect this subregion to shrink onto the critical point as the wall strength is progressively weakened. We note that one can view the wetting line in the $n^{0}-T$ plane as the analog of the wetting temperature isotherm $T=T_{w}$ (a horizontal line) in the $n-T$ phase diagram of a monodisperse system. The variation of daughter distributions with parent density in a polydisperse system causes this line to 'tilt'. The simulation results confirm our heuristic arguments to this effect based on a simple bidisperse example.

Finally, with regard to the wider impact of our findings, we expect these to be relevant to the surface phase behavior of all complex fluids that exhibit significant polydispersity. More specifically, the possibility of density driven wetting transitions could aid an understanding of the factors controlling the morphology of spincoated or ink-jet printed thin films, which arises from the interplay of wetting and phase separation [10]. During these production processes, the solvent is progressively removed, thereby increasing the solute density, and possibly leading to the type of wetting transitions we have described.
[1] R. G. Larson, The Structure and Rheology of Complex fluids (Oxford University Press, New York, 1999).

[2] G. H. Fredrickson, Nature (London) 395, 323 (1998).

[3] For a review, see P. Sollich, J. Phys. Condens. Matter 14, R79 (2002).

[4] K. Ghosh and M. Muthukumar, Phys. Rev. Lett. 91, 158303 (2003).

[5] P. Bartlett and P. B. Warren, Phys. Rev. Lett. 82, 1979 (1999).

[6] N. B. Wilding et al, Phys. Rev. Lett. 95, 155701 (2005); N. B. Wilding et al, J. Chem. Phys. 125, 014908 (2006).

[7] W. K. Wijting, N. A. M. Besseling and M. A. Cohen Stuart, Phys. Rev. Lett. 90, 196101 (2003).

[8] D. G. A. L. Aarts et al, J. Chem. Phys. 120, 1973 (2004).

[9] J. Forsman and C. E. Woodward, Phys. Rev. Lett. 94, 118301 (2005)

[10] S. Y. Heriot and R. A. L. Jones, Nature Materials, 4, 782 (2005); B.-J. de Gans and U.S. Schubert, Langmuir 20, 7789 (2004).

[11] M. Buzzacchi et al, Phys. Rev. E73, 046110 (2006).

[12] We assume that species 2 (taken as the larger particles) has stronger attractive interactions and so phase separates on its own (vertical axis) at the chosen $T$ while the pure species 1 fluid (horizontal axis) does not. With interaction strengths chosen in this way, species 2 particles will typically accumulate in the liquid, with its shorter interparticle distances. The tielines in the sketch therefore cross any dilution line from below, moving from smaller values of $\rho_{2} / \rho_{1}$ at the gas end to larger ones for the coexisting liquid.

[13] For a review of wetting, see eg. S. Dietrich, Phase Transitions and Critical Phenomena, edited by C. Domb and J.L. Lebowitz (Academic, London, 1988), Vol 12.

[14] See eg. R. Kita et al, Phys. Rev. E 55, 3159 (1997).

[15] L. G. MacDowell and M. Müller, J. Chem. Phys. 124, 084907 (2006).

[16] M. Müller and K. Binder, Macromolecules 31, 8323 (1998).

[17] B.A. Berg and T. Neuhaus, Phys. Rev. Lett. 68, 9 (1992).

[18] E.H. Hauge and M. Schick, Phys. Rev. B27, 4288 (1983). 